\title{
Carboxypeptidase $\mathbf{E}$ transmits its anti-migratory function in glioma cells via transcriptional regulation of cell architecture and motility regulating factors
}

\author{
ANGELA ARMENTO $^{1}$, ELENA I. ILINA ${ }^{2,3}$, TONY KAOMA ${ }^{4}$, ARNAUD MULLER $^{4}$, LAURENT VALLAR $^{4}$, \\ SIMONE P. NICLOU ${ }^{3}$, MARCEL A. KRÜGER ${ }^{5}$, MICHEL MITTELBRONN $^{2,3,6-8}$ and ULRIKE NAUMANN ${ }^{1}$
}

\begin{abstract}
${ }^{1}$ Molecular Neuro-Oncology, Hertie Institute for Clinical Brain Research and Center Neurology, University of Tübingen, D-72076 Tübingen, Germany; ${ }^{2}$ Luxembourg Centre of Neuropathology (LCNP), L-3555 Dudelange; ${ }^{3}$ NorLux NeuroOncology Laboratory, Luxembourg Institute of Health, L-1526 Luxembourg; ${ }^{4}$ Genomics and Proteomics Research Unit, Department of Oncology, Luxembourg Institute of Health (L.I.H.), L-1526 Luxembourg, Luxembourg; ${ }^{5}$ Department of Preclinical Imaging and Radiopharmacy, University of Tübingen, D-72076 Tübingen; ${ }^{6}$ Neurological Institute (Edinger

Institute), Goethe University Frankfurt, D-60528 Frankfurt/Main, Germany; ${ }^{7}$ Laboratoire Nationale de Santé, Dudelange, L-3555 Dudelange; ${ }^{8}$ Luxembourg Centre for Systems Biomedicine (LCSB),

University of Luxembourg, L-4362 Esch-sur-Alzette, Luxembourg
\end{abstract}

Received March 15, 2017; Accepted June 6, 2017

DOI: $10.3892 /$ ijo.2017.4051

\begin{abstract}
Glioblastoma (GBM), the most frequent and aggressive malignant primary brain tumor, is characterized by a highly invasive growth. In our previous study we showed that overexpression of Carboxypeptidase E (CPE) mitigated glioma cell migration. In the present study we aimed at deciphering the regulatory mechanisms of the secreted form of CPE (sCPE). By transcriptome analysis and inhibition of signaling pathways involved in the regulation of cell growth and motility, we discovered that overexpression of sCPE was accompanied by differential regulation of mRNAs connected to the motility-associated networks, among others FAK, PAK, Cdc42, integrin, STAT3 as well as TGF- $\beta$. Especially SLUG was downregulated in sCPE-overexpressing glioma cells, paralleled by reduced expression of matrix-metalloproteinases (MMP) and, in consequence, by decreased cell migration. Expression of SLUG was regulated by ERK since inhibition of ERK reverted sCPE-mediated SLUG downregulation and enhanced cell motility. In a mouse glioma model, overexpression of sCPE significantly prolonged survival. Our results implicate a novel role for sCPE that mainly affects the expression of motility-associated genes via several signal pathways.
\end{abstract}

Correspondence to: Professor Ulrike Naumann, Molecular Neuro-Oncology, Hertie Institute for Clinical Brain Research and Center Neurology, University of Tübingen, Otfried-Müller-Strasse 27, D-72076 Tübingen, Germany

E-mail: ulrike.naumann@uni-tuebingen.de

Key words: glioma, sCPE, cell motility, SLUG, gene expression

\section{Introduction}

Glioblastoma (GBM) is the most malignant brain tumor in humans and it is characterized by diffuse and infiltrative growth into the surrounding brain tissue, enhanced cell growth, the ability to overcome cell death and antitumor immune responses and therapy resistance. The main reason for the high malignancy of GBM is the highly infiltrative phenotype, which is why complete surgical removal of all tumor cells is impossible and recurrence inevitable. During disease progression a shift toward a more mesenchymal phenotype is common. Especially the mesenchymal subtype of GBM is associated with an aggressive phenotype, and GBM cells specifically use a mesenchymal mode of cell migration (1). Enhanced expression of epithelial to mesenchymal transition (EMT) proteins like SLUG or ZEB2 correlates with an invasive phenotype of GBM, high tumor grade and faster tumor progression $(2,3)$. Beside the involvement of EMT proteins, migration and invasion of glioma cells are regulated by other processes, including changes in cell metabolism, destruction of the extracellular matrix (ECM) and regulation of cell adhesion and cell architecture. Non-coding RNAs, transcription factors, cell surface proteins, secreted cyto- and chemokines and other proteins with different functions are involved in the enhancement of motility of GBM cells (reviewed in ref. 4).

CPE was originally described as a neuropeptide-processing enzyme. Numerous studies have shown that CPE, which is present in different variants that are products of differential splicing, proteolytic cleavage or glycosylation, is a multifunctional protein that plays roles in the endocrine and nervous systems and also in cancer (5). An alternatively spliced CPE transcript $(\triangle \mathrm{N}-\mathrm{CPE})$ has been found in human tumor cells. It encodes a cytoplasmic protein lacking a part of the N-terminal region due to alternative splicing of the first exon. $\triangle \mathrm{N}-\mathrm{CPE}$ 
was described to increase the metastatic potential of tumor cells by upregulating metastasis-related genes (6). We recently identified a secreted version of CPE (sCPE) that, in contrast to $\triangle \mathrm{N}-\mathrm{CPE}$, provides an anti-migratory and anti-invasive, but pro-proliferative function in glioma cells. sCPE is released into the extracellular space, where it, at least partially by modulating the cell architecture and increasing cell adhesion, transmits its anti-migratory function (7).

One key signaling element in the malignant behavior of glioma is its high invasive phenotype which involves the stimulation of a variety of motility-associated signaling cascades. Modulation of these cascades by external and internal stimuli results into changes in gene expression and in the execution of glioma cell migration and invasion. While it has been described that in hippocampal neurons CPE induces the expression of the known pro-invasive and anti-apopotic $B$-cell lymphoma 2 (BCL-2) protein via ERK1/2 signaling $(8,9)$, information on additional mechanisms, pathways or factors by which sCPE provides its anti-migratory effect in glioma cells are still rare. The aim of the present study therefore, was to identify targets of sCPE as well as signaling cascades that are affected by SCPE and that are specific for transmitting its anti-migratory effects in glioma cells.

Our findings show that sCPE modifies the expression of a panel of mRNAs involved in the modulation of migration, invasion and cell architecture or that are part of motility-associated signaling cascades integrating the transforming growth factor beta (TGF- $\beta$ ), the integrin, the focal adhesion kinase (FAK), the cell division cycle 42 (Cdc42), the p21-activated kinase (PAK)-1 as well as the signal transducer and activator of transcription 3 (STAT3) and the mitogen-activated protein kinase (MAPK/ERK) pathways. One prominent factor that was downregulated in sCPE-overexpressing glioma cells was the oncogenic protein SLUG that has been described as an invasion-related transcription factor overexpressed in many cancers including glioma (10). In our in vitro cell culture model reduced SLUG expression mediated by SCPE was, at least partially, responsible for the observed anti-migratory phenotype of sCPE-overexpressing glioma cells.

\section{Materials and methods}

Cells, viruses and reagents. LNT-229 and LN-308 human malignant glioma cells were kindly provided by N. de Tribolet (Lausanne, Switzerland), T98G cells were purchased from the American Type Culture Collection (ATCC; Manassas, VA, USA). Tu-132 and Tu-140 GBM primary cells were established from human GBM tissue and used at passages 5-10. The use of primary glioma cells was approved by the ethics committee of the University of Tübingen (125/2007BO1). All cells were maintained in Dulbecco's modified Eagle's medium (DMEM; Gibco-Life Technologies, Eggenstein, Germany) containing $10 \%$ fetal calf serum (FCS; Gibco), penicillin (100 U/ml), streptomycin $(100 \mu \mathrm{g} / \mathrm{ml})$ and the appropriate selection antibiotic in a humidified atmosphere containing $5 \% \mathrm{CO}_{2}$. The generation of sCPE-overexpressing (LNT-229-rCPE, from Rattus norvegicus) or neo control cells (LNT-229-neo) has been previously described (7). Besides this, stable glioma cell lines overexpressing human CPE under control of the CMV promoter were generated by transduction of the cells with the lentivirus pReceiver LV105-CPE or its empty counterpart (GeneCopeia, Inc., Rockville, MD, USA) followed by the selection with puromycin. Rat and human mature CPE protein shows $96 \%$ identity and $98 \%$ similarity (5) in the amino acid (AA) sequence and a total conservation of the Zn-carboxypeptidase domain, indicating that the enzymatic activity is not different between the species. Besides, a correct procession and maturation of $\mathrm{SCPE}$ is conserved in both species, since the penta-arginine sequence $\left(R R R R R_{42}\right)$ is identical. Changes are present in the AA sequence in closest distance to the prohormone sorting signal binding site (the region between $\mathrm{R}_{255}$ and $\mathrm{K}_{260}$ ). To avoid 'off target' effects, CPE-knockdown in Tu-140 cells was induced by transient transfection with CPE-specific or non-target POOL siRNA (siTOOLs Biotech $\mathrm{GmbH}$, Martinsried, Germany) and Lipofectamine 2000 (Thermo Fisher Scientific, Waltham, MA, USA) or by lentiviral transduction using pGIPZ vectors carrying either control or CPE-specific shRNA. The SLUG knockdown in LNT-229, LN-308, T98G and Tu-132 cells was generated by transfection of the cells with SLUG-specific or control POOL siRNA (MISSON esiRNA huSNAI2; MISSION esiRNA EGFP; Sigma-Aldrich, Darmstadt, Germany) using the Viromer BLUE transfection kit (Biozym Scientific GmbH, Hessisch Oldendorf, Germany). The construction of Ad-EGFP has been described (11). For the generation of Ad-SLUG, human SLUG cDNA was cloned into pTRACK-CMV using the Ad-Easy system provided by B. Vogelstein (Baltimore, MD, USA) (12). Ad-SLUG additionally codes for EGFP in a second expression cassette. Recombinant adenoviral genomes were transfected into HEK-293 cells (ATCC). Viruses were CsCl-purified, dialysed and titrated using the Clontech Rapid Titration system (Takara Bio Europe SAS, Saint-Germain-en-Laye, France). Transgene expression was verified by immunoblot. Infection with recombinant adenoviruses was accomplished by exposing cells to $100 \mathrm{MOI}$ of the appropriate adenovirus in serum-free DMEM for 15 min followed by the addition of serum-containing medium. To inhibit ERK1/2 activity, glioma cells were treated with the MAPK inhibitor U0126 (10 $\mu \mathrm{M}, 24$ h; R\&D Systems GmbH, Wiesbaden, Germany). If not mentioned otherwise, all other reagents were from Sigma-Aldrich.

Measurement of cell migration. The Transwell migration assay have been previously described (7). Briefly, cells were seeded in the upper layer of $8 \mu \mathrm{m}$ pore-sized Boyden Trans well chambers and were allowed to actively migrate for $18 \mathrm{~h}$ towards FCS containing DMEM as attractant medium placed in the bottom chamber. Migrated cells on the lower layer of the membrane were fixed, stained with hematoxylin/eosin and counted. Number of migrated cells was normalized to cell density at the end of the migration period, assessed in parallel by crystal violet staining as previously described (13).

Immunofluorescence. For the assessment of cytoskeleton changes, the cells were seeded on poly-L-lysin coated coverslips, allowed to grow and fixed in $4.5 \%$ formaldehyde. Staining was accomplished using the Actin Cytoskeleton and Focal Adhesion Staining kit (Millipore, Schwalbach, Germany) which consists of TRITC-conjugated phalloidin allowing the detection of filamentous actin in the cytoskeleton, 
anti-vinculin as a universal focal adhesion marker and DAPI to stain the nucleus. Quantification of cell adhesion was done by measuring the vinculin positive area using the ImageJ software (National Institute of Health, Bethesda, MD, USA) and counting the number of large focal adhesion complexes (LFA) per cell.

Immunoblot analysis and detection of receptor phosphorylation. The general immunoblot procedure has been described elsewhere (11). For the detection of phospho-EGFR, LNT-229 cells were serum starved for $24 \mathrm{~h}$ followed by the addition of conditioned medium produced by either LNT-229 control or CPE-overexpressing cells. For generation of supernatants/conditioned medium, the cells were treated as indicated and serum-free medium was added. Supernatants were harvested 24 or $48 \mathrm{~h}$ later, clarified from cell debris by centrifugation and concentrated using Amicon concentrators (Millipore). Protein contents were analyzed according to Bradford. Lysates collected for the detection of receptor phosphorylation were lysed in RIPA buffer and protein determination was performed using the BCA assay (Pierce BCA protein assay kit; Thermo Fisher Scientific, Darmstadt, Germany) according to the manufacturer's protocol. Following antibodies were used: CPE (\#610758, 1:2,000; BD Biosciences, Heidelberg, Germany), MT1-MMP/MMP14 (\#2010-1, 1:1,000; Epitomics Burlingame CA, USA) and TIMP-2 (Mab971, 1:1,000; R\&D Systems). SLUG (\#9585, 1:1,000), MMP-2 (\#4022, 1:1,000), the phospho-EGFR antibody sampler kit (\#9922, 1:1,000), the PathScan Multiplex Western Cocktail I (for the detection of phospho-ERK1/2,\#5301, 1:1,000), STAT3 (\#9139, 1:2,000) and phospho-STAT3 ${ }^{\text {S727 }}$ (\#9134, 1:1,000) were purchased from Cell Signaling Technology (Frankfurt am Main, Germany). ERK1/2 (sc-135900, 1:2,000), Bcl-2 (sc-509, 1:1,000) and $\alpha$-tubulin (sc-12462-R, 1:2,000) were from Santa Cruz Biotechnology (Heidelberg, Germany). Actin was provided by Abcam (ab8227, 1:2,000; Cambridge, UK) and GAPDH from Chemicon (Billerica, MA, USA; AB2302, 1:2,000). Protein expression was quantified using the ChemiDoc MP system and ImageLab 5.1 software (Bio-Rad Laboratories, Munich, Germany). For normalization of protein expression in lysates, either GAPDH or $\alpha$-tubulin was used as indicated. For normalization of protein expression in supernatants, the membranes were stained with Ponceau S and total stained protein was used. For the detection of phosphorylated receptors, the receptor tyrosine kinase phosphorylation array (Human Phospho-Receptor Tyrosine Kinase Array kit; R\&D Systems) was executed according to the manufacturer's protocol.

Transcriptome profiling experiments. Total RNAs were extracted using the standard TRIzol (Invitrogen) protocol provided by the manufacturer for preparation of mRNA. RNA purity and integrity were monitored using NanoDrop ${ }^{\circledR}$ ND-1000 spectrophotometer and Agilent 2100 Bioanalyzer with RNA 6000 Nano assay kit. Only RNAs with no sign of contamination or marked degradation $(\mathrm{RIN}>9)$ were considered good quality and used for further analysis. Transcriptome profiles were determined in the same triplicate of RNAs using the Affymetrix Human Transcriptome Array 2.0. For whole-transcript expression analysis, $100 \mathrm{ng}$ of total RNAs were processed and labeled using the GeneChip WT PLUS Reagent kit (Affymetrix, Santa Clara, CA, USA). Upon hybridization of labelled products, arrays were washed and stained using the Affymetrix GeneChip WT Terminal Labeling and Hybridization kit, before being scanned using a GeneChip Scanner 3000.

Microarray data analysis. CEL files generated upon array scanning were imported into Partek ${ }^{\circledR}$ Genomics Suite $^{\mathrm{TM}}$ (GS) 6.6 for preprocessing. Partek was set up to run standard RMA at the probeset level. Resulting $\log 2$ probeset intensities were then imported into R statistical environment (http://www.R-project. org/) for further analysis. First, $\log 2$ intensity values were summarized to estimate the expression level of each transcript cluster (TC) by averaging the intensity signals from the corresponding probeset regions. Matching between probesets, TCs and targeted genes was verified through Affymetrix annotation files (HTA-2_0 probeset and transcript hg19 na33.1 csv file). The quality of the data was then evaluated by assessing repeatability Pearson's correlation coefficients, and through visual inspection of density plots and relative log expression plots. Principal component analysis was also used to reduce dimensionality of the data, visualize the concordance between biological replicates, and assess if the variability in data actually reflected what was expected from the experimental design. Finally, the Limma package (R/Bioconductor) was used to estimate the statistical significance of TC expression level differences between LNT-229-rCPE and LNT-229-neo samples as the reference. Resulting P-values were adjusted for multiple testing error using the Benjamini and Hochberg's false discovery rate (FDR) (14). Elements with a FDR $<0.05$ were considered as differentially expressed (DE), irrespective of the fold-change. Microarray expression data are available at ArrayExpress (http://www.ebi.ac.uk/arrayexpress) under the accession number E-MTAB-5297. The Qiagen's Ingenuity ${ }^{\circledR}$ Pathway Analysis software (IPA; Qiagen Redwood City, Inc., Redwood City, CA, USA; www.qiagen.com/ingenuity) was used for transcript cluster mapping and for data mining, including functional analyses, upstream analysis and gene network reconstruction. Heatmaps were generated corresponding to significant genes with an FDR of $<0.01$. Right-tailed Fisher's exact test was used to calculate a P-value for functional enrichment analysis (threshold: $-\log (\mathrm{P}$-value) $>1.301$, corresponding to a P-value $<0.05$ ).

Quantitative RT-PCR. RNA was reverse-transcribed using SuperScript II (Invitrogen GmbH, Karlsruhe, Germany). Target gene expression was determined using the SYBR-Green Master Mix (Thermo Fisher Scientific) on an ABI 7200 system. Relative mRNA expression was quantified ([ $\mathrm{E}^{\mathrm{DCT}}$ (gene of interest)/ $\mathrm{E}^{\mathrm{DCT}}$ (housekeeping gene)]). The following primers were used: huCPE-f, CCACCATGTCGCAAGAAT GA and huCPE-r, AAGCTCCACGGTGATCTCAAA; rCPE-f, ATGGGAATGAGGCTGTTGGAC and rCPE-r, GG CATGATGTGAATGCGGGTA; RPLP0-f, GAGTCCTGGC CTTGTCTGTGG and RPLP0-r, TCCGACTCTTCCTTG GCTTCA; SPP1-f, GCCGAGGTGATAGTGTGGTT and SPP1-r, ACGGCTGTCCCAATCAGAAG; SLUG-f, CATAC CACAACCAGAGATCC and SLUG-r, GAGGAGTATCCGG AAAGAGG; STC1-f, AAGATGGCGACCACCAAAGT and 


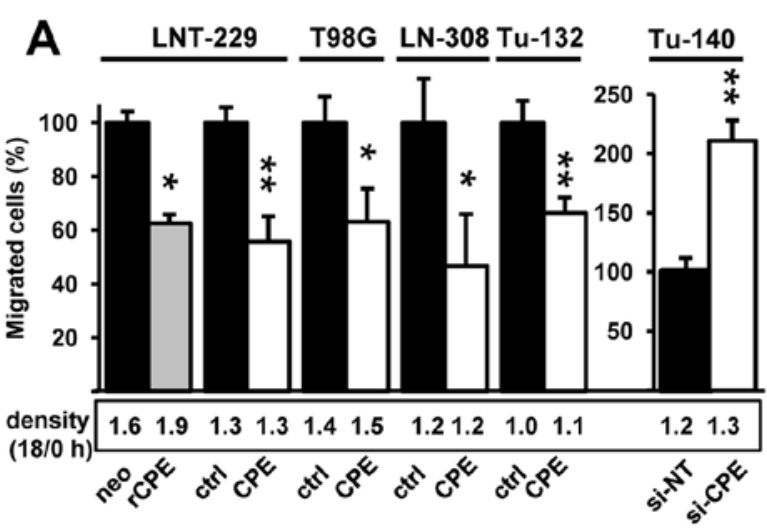

B
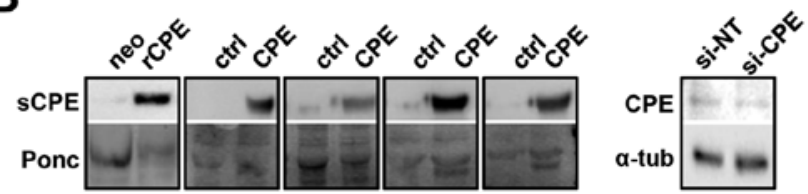
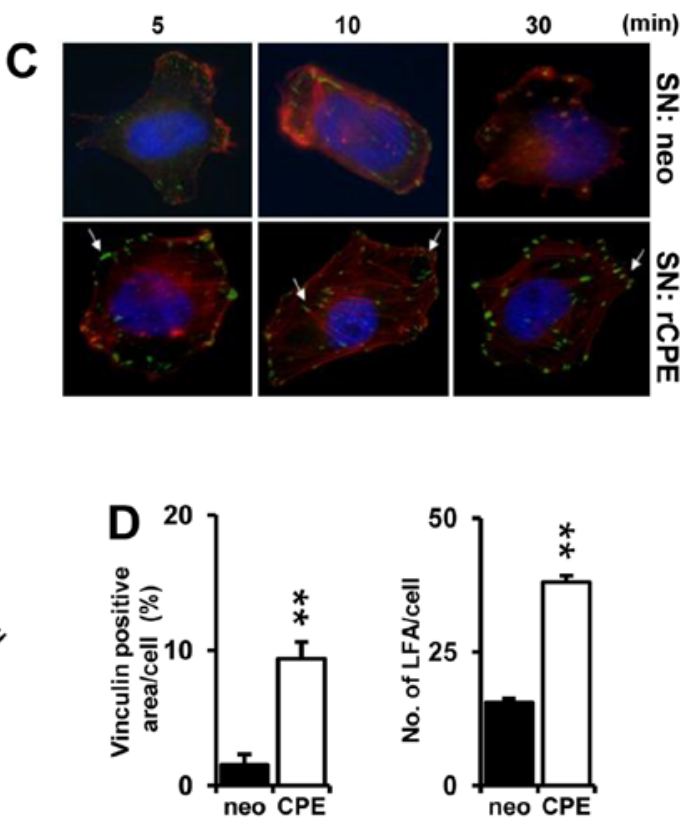

Figure 1. sCPE mitigates migration and adhesion in glioma cells. (A) Cell migration was analyzed by Transwell migration assay in LNT-229, T98G, LN-308 and Tu-132 control (black bars), rat sCPE- (grey bar) or human sCPE (white bars) overexpressing cells (left panel, n=3, SEM), or in CPE-knockdown Tu-140 cells $48 \mathrm{~h}$ after siRNA transfection with either siCPE (white bar) or non-target (NT) siRNA (black bar; right panel; $\mathrm{n}=3$, SEM). Values under the bars determine cell density at the end of the migration compared to the seeding time. Migration values were normalized to cell density. (B) Immunoblot presenting the expression of CPE in cell supernatants (left panel) or lysates (right panel). (C) Immunofluorescence staining of vinculin (green) and filamentous actin (red) of LNT-229 cells treated with supernatant from either LNT-229-rCPE or LNT-229-neo control cells for the indicated time periods (magnification, x63). Arrows indicated the formation of LFA complexes. (D) Quantification of the vinculin positive area (left panel) and the total number of LFA (right panel) in LNT-229 cells treated for 30 min with supernatants generated from different LNT-229-neo- or sCPE-overexpressing cell clones (SD).

STC1-r, GCAGTGACGCTCATAAGGGA; MGST1-f, GG TTTTGTTTATGGTACTTCAGAGT and MGST1-r, TGTG AATTGTTCATTTAGATGTGCC; CD9-f, AAACGCTGAA AGCCATCCAC and CD9-r, GATGGCATCAGGACAGGAC TT; MGAT4A-f, TGGTGTTGCAGAAGGAATGGT and MGAT4A-r, TCAGATGATCAGTTGGTGGCT; ADAMTS4-f, GACAAGTGCATGGTGTGCG and ADAMTS4-r, GCCGG ACAAGAATGTGGGT; PCDH17-f, AGTTTGTTCAAAGTA GCTCCACG and PCDH17-r, TCACAGCAGGAGCCTTTG TT. RPLP0 primers were used for normalization.

Mouse experiments. Athymic FoxN1-deficient NMRI nude mice were purchased from Janvier Labs (Saint Berthevin, France). Deficiency in the FoxN1 gene provides thymic aplasia which results in immunodeficiency regarding lack of T cells, whereas $\mathrm{B}$ and $\mathrm{NK}$ cells remain. The mutation also leads to a defect of the hair follicule leading to transient downy hair. When this duvet is gone, mice appear nude. Female mice of 5 weeks of age were used in all experiments. Mice were held in groups of 4 to 5 mice in filter top cages under sterile conditions in the animal facility of the Hertie Institute at a temperature of $24^{\circ} \mathrm{C}$ and $50 \%$ humidity according to the regulations of the Society for Laboratory Animal Science. For tumor growth, mice were anaesthetized using fentanyl, midazolam and medetomidin and 100,000 cells were implanted in the right striatum of the mice. After surgery, anesthesia was neutralized using naloxon, flumazenil and atipazemol. Analgetics (carpofen) were added intraoperatively. The mice were sacrified when neurological symptoms like tremor, tilt of the head, hemiparesis, hemiplegia or uncoordinated motion appeared of if the mice lost weight (fixed end-point experiment). Tumor burden was determined optically. Survival analyses were done by generating Kaplan-Meier survival curves. The animal experiments were licensed by the regional board Tübingen and were performed according to the German law, Guide for Care and Use of Laboratory Animals.

Statistical analysis. The figures show data obtained in at least three independent experiments as indicated. Statistical analyses were performed using GraphPad Prism version 6.0, (GraphPad Software, Inc., La Jolla, CA, USA). Quantitative data were assessed for significance by t-test $(\mathrm{P}<0.05 ; \mathrm{P}<0.01$; $\mathrm{P}<0.001)$. Survival of mice was analyzed by Kaplan-Meier live table and for comparison of survival, the Wilcoxon and log-rank tests were used (significance level a=0.05; JMP 11.0 software; SAS Institute, Inc., Cary, NC, USA).

\section{Results}

sCPE mitigates cell motility and enhances cell adhesion in established and primary glioma cells. We have previously shown that sCPE reduced cell motility in LNT-229 and LN-308 glioma cell lines (7). To demonstrate a general anti-migratory function of sCPE in glioma, we generated additional sCPE-overexpressing established and a primary low passage glioma cell lines by lentiviral transduction using pReceiver LV105-CPE. Overexpression of both human or rat SCPE mitigates migration in glioma cell lines (LNT-229, T98G and LN-308) and primary low passage Tu-132 glioma cells. In comparison and as demonstrated before for LNT-229 cells (7), the knockdown of sCPE induces cell motility in Tu-140 that possess higher endogenous CPE levels compared 

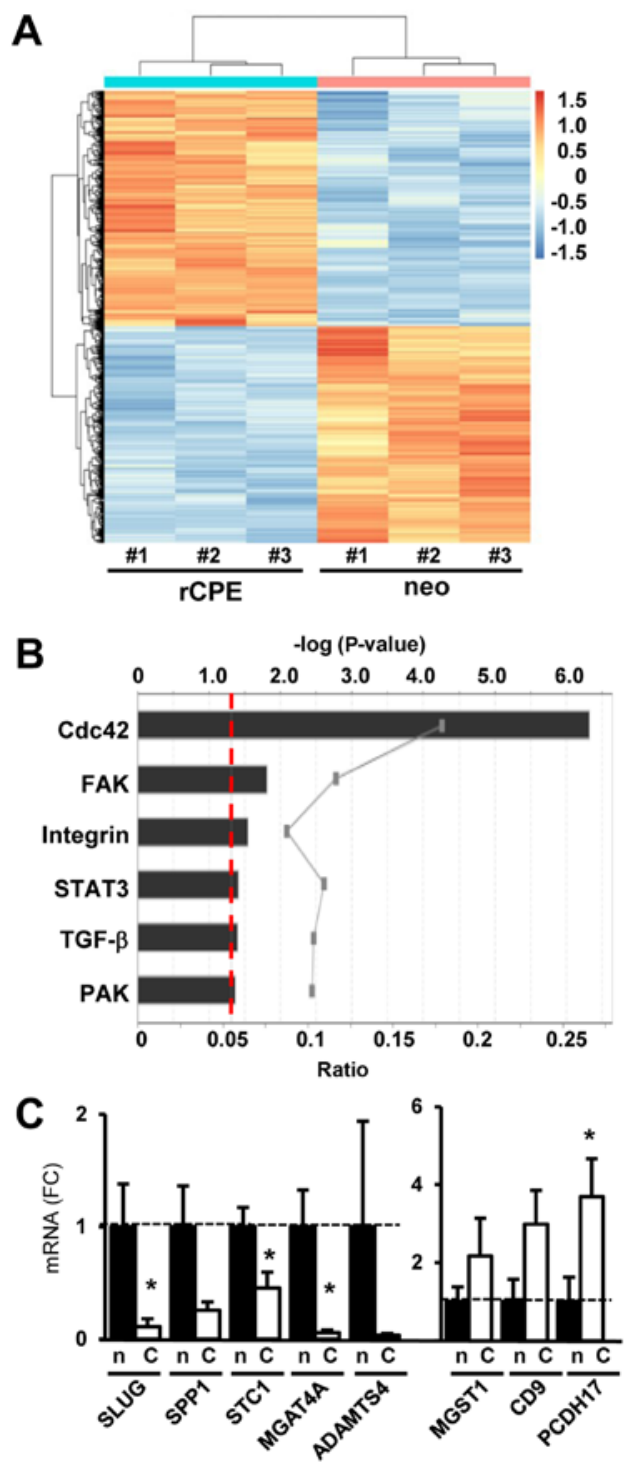

Figure 2. sCPE serves as a regulator for the expression of genes, associated with prominent motility signaling networks. (A) Heatmap of differentially expressed genes (FDR <0.01). (B) IPA-based analysis of differential gene expression and its association to signaling networks representing enriched canonical pathways, considered as important in cell motility. The red dashed line indicates the threshold. (C) qRT-PCR based validation of the expression of assorted genes associated with cell mobility and cell architecture that were found to be differentially regulated in LNT-229-neo (n) vs. LNT-229-rCPE (C) cells by mRNA microarray data. Validation was performed in at least three different sCPE-overexpressing and neo control LNT-229 clonal cell lines $(n>3, S E M)$.

to Tu-132 cells (Fig. 1A and B). According to the 'Grow or Go' hypothesis, proliferation and migration are inversely regulated (15). For this reason and to avoid that migration rates were influenced by proliferation, we also measured cell density at the start and end of the migration period, and also for longer time periods. Only rat CPE overexpressing LNT-229 cells showed slightly elevated proliferation [(7) and data not shown]. Even if the differences in cell density were negligible at the end of the migration period (Fig. 1A, lower panel) we used these values to normalize cell migration. We have previously shown that overexpression of CPE induced the generation of large focal adhesion complexes (LFA) in LNT-229 cells suggesting a more adhesive phenotype (7). This effect is most
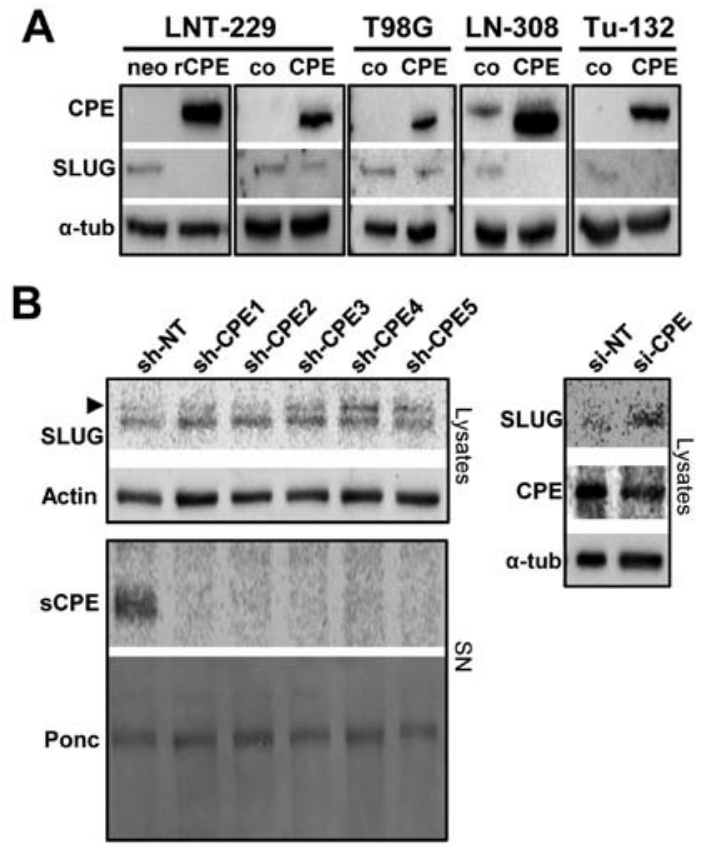

Figure 3. sCPE mediates the expression of SLUG. (A) Immunoblot of SLUG expression in neo, control (co) or sCPE-overexpressing glioma cell lines. $\alpha$-tubulin ( $\alpha$-tub) was used as a loading control (one representative experiment is shown, $n=3$ ). (B) Immunoblot of SLUG expression in pGZIP-sh-CPE lentivirally transduced (left panel) or CPE-siRNA transiently transfected (right panel) Tu-140 cells. Detection of actin, $\alpha$-tubulin or Ponceau S staining were used as loading controls (one representative experiment is shown).

probably mediated by SCPE since addition of SCPE-containing supernatants induced the formation of vinculin-positive LFA and actin stress fibers in LNT-229 cells (Fig. 1C and D).

SCPE affects the expression of genes mostly related to cell architecture and motility. Considering that extracellular sCPE could serve as a ligand or signaling factor, or might compete with the binding of extracellular ligands to their receptors, finally leading to changes in gene expression, we were interested whether overexpression of SCPE may alter the expression of motility-associated genes in glioma cells. We therefore analyzed differential gene expression in less migratory sCPE-overexpressing LNT-229 (LNT-229-rCPE) and its sibling cell line (LNT-229-neo) that migrates faster and secrets only a very low amount of sCPE (Fig. 1A). Using mRNA expression data, we found 1065 mRNAs with a false discovery rate (FDR) $<0.01$ to be differentially expressed in $\mathrm{SCPE}$-overexpressing compared to neo control cells. In this group a heatmap plot represents the intensity of significant genes (FDR $<0.01$ ) deciphering that the two cell lines properly cluster in a balanced number of upregulated and downregulated genes (Fig. 2A). IPA, further detailed analyses and a broad literature search showed that in the panel of these differentially expressed genes at least 100 genes were connected to the regulation of cell motility. Besides, IPA demonstrated an enrichment of differentially expressed mRNAs in sCPE-overexpressing LNT-229 cells that are associated to the Cdc42-, FAK-, STAT3-, TGF- $\beta$-, PAK- and integrin-signaling pathways (Fig. 2B and Table I), with a tendency to a reduced activation of the Cdc42-, TGF- $\beta$-, PAK- and integrin-signaling pathways in these cells (data not shown). After activation, these pathways are known to induce cell motility. 


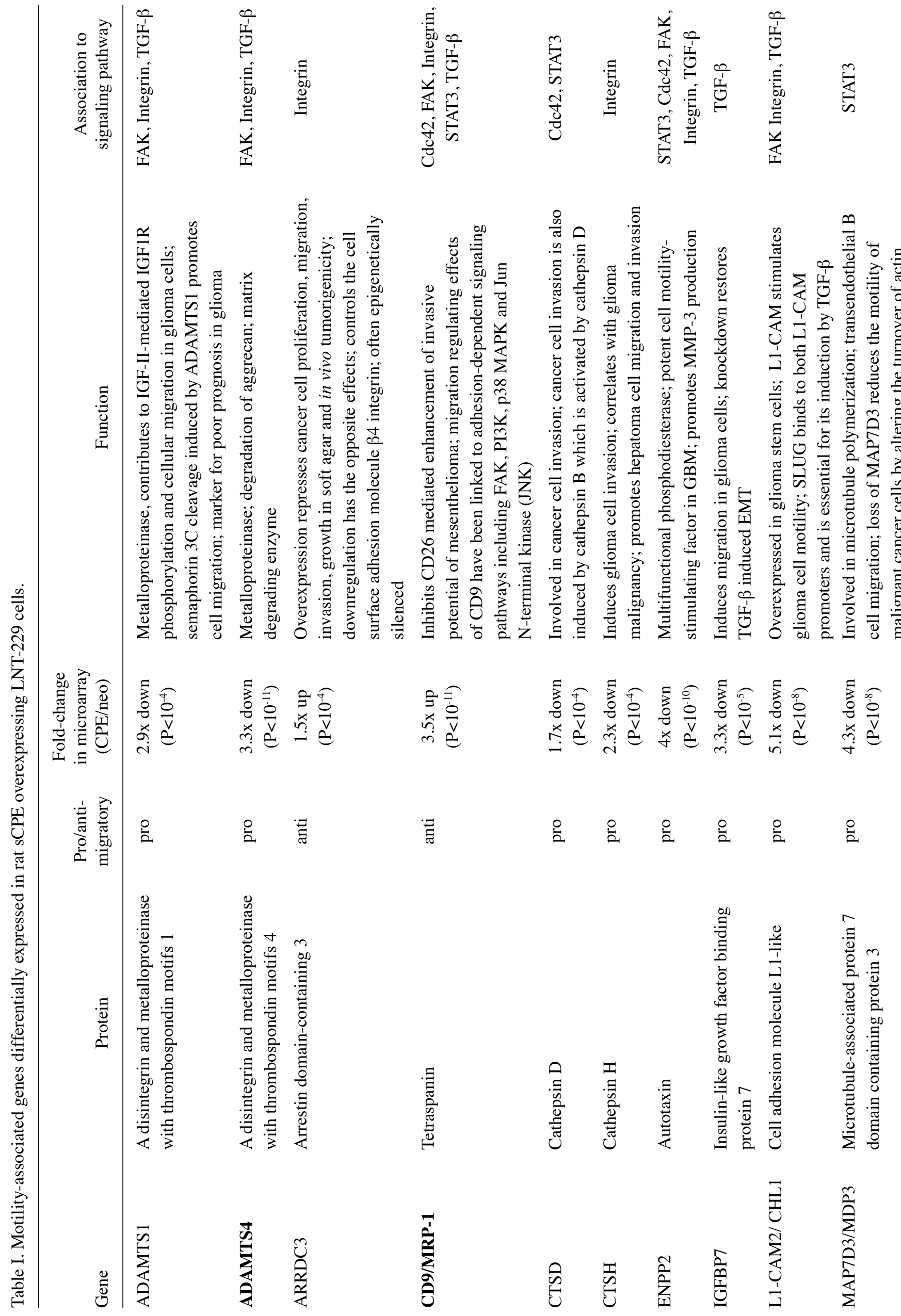




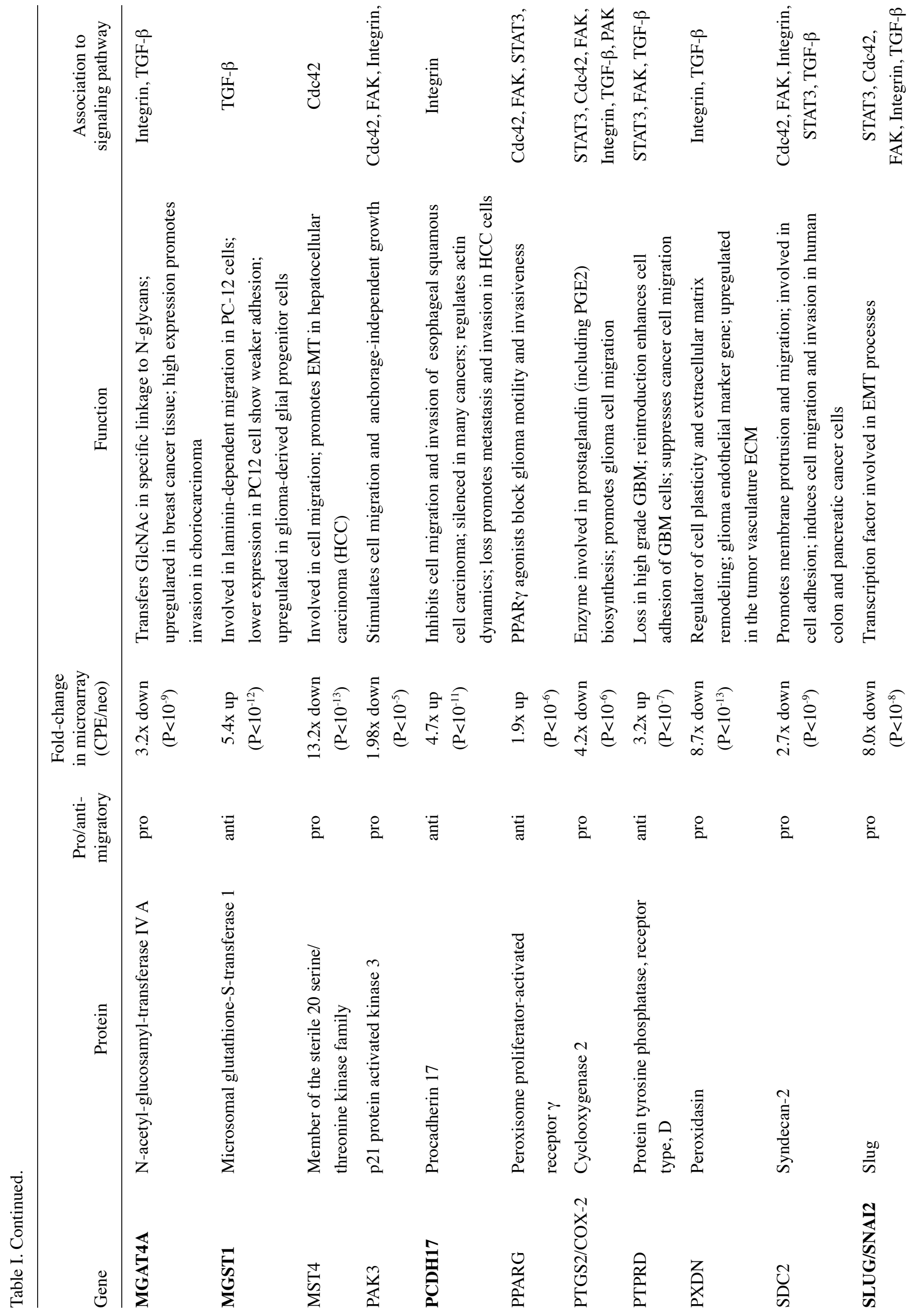



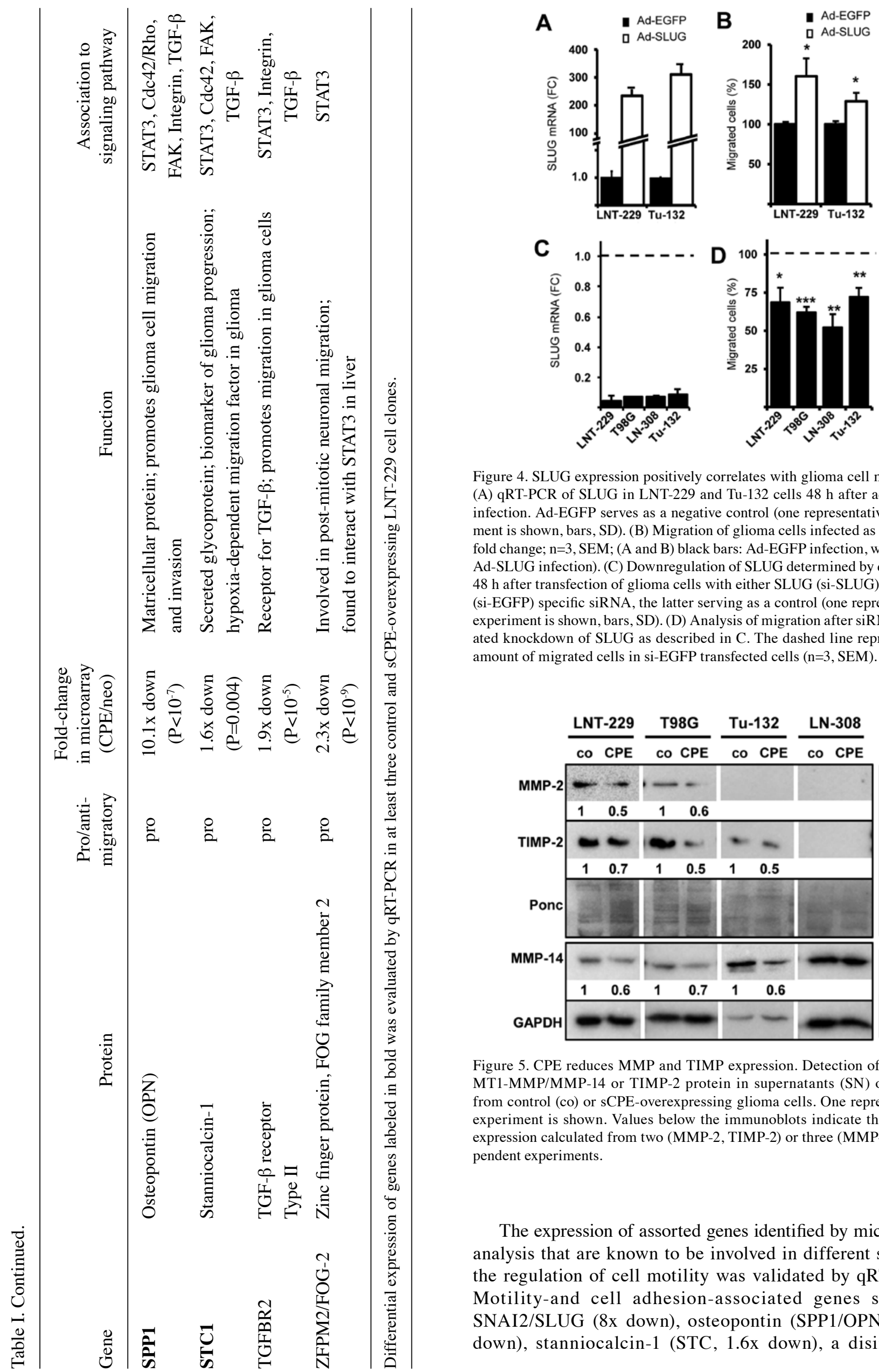

Figure 4. SLUG expression positively correlates with glioma cell migration. (A) qRT-PCR of SLUG in LNT-229 and Tu-132 cells $48 \mathrm{~h}$ after adenoviral infection. Ad-EGFP serves as a negative control (one representative experiment is shown, bars, SD). (B) Migration of glioma cells infected as in A (FC, fold change; $n=3$, SEM; (A and B) black bars: Ad-EGFP infection, white bars: Ad-SLUG infection). (C) Downregulation of SLUG determined by qRT-PCR $48 \mathrm{~h}$ after transfection of glioma cells with either SLUG (si-SLUG) or EGFP (si-EGFP) specific siRNA, the latter serving as a control (one representative experiment is shown, bars, SD). (D) Analysis of migration after siRNA mediated knockdown of SLUG as described in C. The dashed line represent the amount of migrated cells in si-EGFP transfected cells $(n=3, S E M)$.

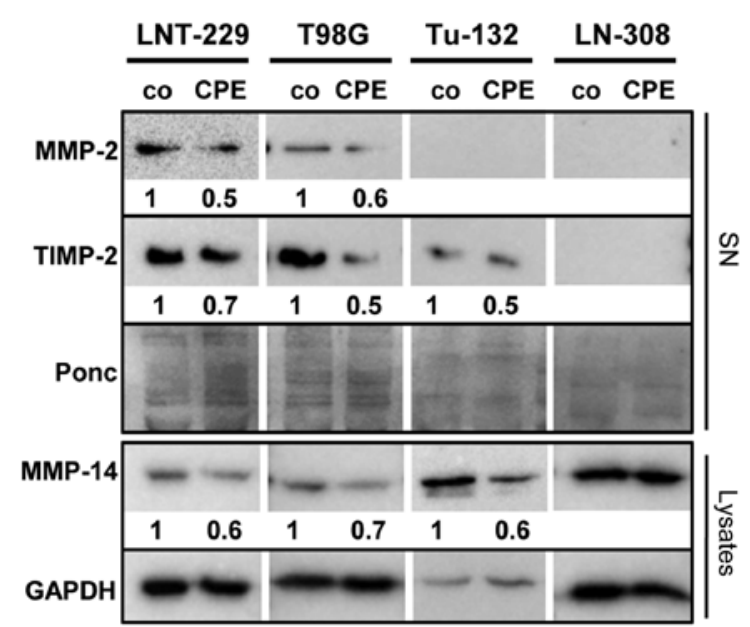

Figure 5. CPE reduces MMP and TIMP expression. Detection of MMP-2, MT1-MMP/MMP-14 or TIMP-2 protein in supernatants (SN) or lysates from control (co) or sCPE-overexpressing glioma cells. One representative experiment is shown. Values below the immunoblots indicate the relative expression calculated from two (MMP-2, TIMP-2) or three (MMP-14) independent experiments.

The expression of assorted genes identified by microarray analysis that are known to be involved in different steps of the regulation of cell motility was validated by qRT-PCR. Motility-and cell adhesion-associated genes such as SNAI2/SLUG (8x down), osteopontin (SPP1/OPN, 10.1x down), stanniocalcin-1 (STC, 1.6x down), a disintegrin 

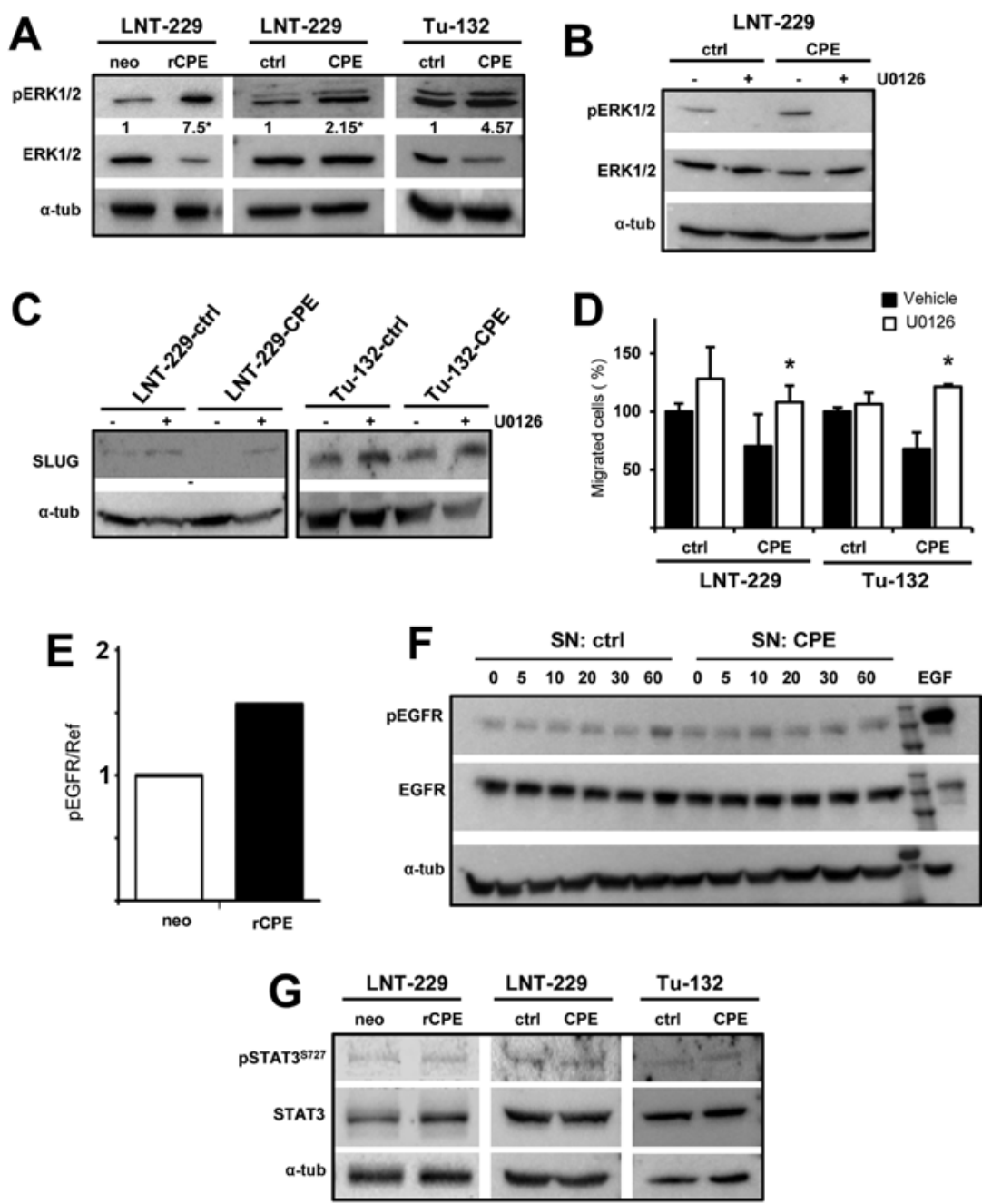

Figure 6. Inhibition of ERK abolishes sCPE-mediated SLUG downregulation and induces cell migration. (A) Determination of phospho-ERK1/2 (P-ERK1/2) in LNT-229 and Tu-132 sCPE-overexpressing and control cells. Values indicate the fold-change of phospho-ERK1/2 to ERK1/2 signal density (n=3, one representative immunoblot is shown). (B) Inhibition of phospho-ERK1/2 by treatment of LNT-229 glioma cells with U0126. (C) SLUG protein expression in control or sCPE-overexpressing LNT-229 and Tu-132 cells cultivated without or with U0126 $(10 \mu \mathrm{M})$ for $24 \mathrm{~h}(\mathrm{n}=3$, one representative experiment is shown). (D) Migration of control or sCPE-overexpressing LNT-229 and Tu-132 cells treated as indicated in C ( $\mathrm{n}=3$, SEM). (E) EGFR-phosphorylation determined by membrane-based array for the detection of receptor tyrosine kinase phosphorylation in rat sCPE-overexpressing LNT-229 cells. (F) Immunoblot of serumdeprived LNT-229 cells cultivated for the indicated time periods in sCPE-containing conditioned medium. As a positive control the cells were treated with recombinant human EGF $(10 \mathrm{ng} / \mathrm{ml}, 30 \mathrm{~min})$. ( $\mathrm{n}=2$, one representative experiment is shown). (G) Phospho-STAT3S727 was determined by immunoblot of sCPE-overexpressing LNT-229 and Tu-132 cells. ( $\mathrm{n}=3$, one representative experiment is shown).

and metalloprotease with thrombospondin motifs (ADAMTS4, 3.3x down), procaherin-17 (PCDH17, 4.7x up), tetraspanin/motility related protein-1 (CD9/MRP-1, 3.5x up), $\mathrm{N}$-acetyl-glucosamyl-transferase IV A (MGAT4A, 5.4x down), microsomal glutathione-S-transferase 1 (MGST1, 4.2x down) and others were differently expressed in sCPE-overexpressing compared to neo control LNT-229 cells (Table I and Fig. 2C).

SLUG expression negatively correlates with $S C P E$ and glioma cell migration. Since we found SLUG as one prominent factor being downregulated in rat sCPE-overexpressing LNT-229 cells, we tested whether overexpression of human sCPE provides the same effect. Indeed, reduced expression of SLUG was detected in all human sCPE-overexpressing low passage primary and established glioma cell lines (Fig. 3A). Vice versa, knockdown of CPE in Tu-140 cells that provide elevated CPE secretion but no basal SLUG, induced the expression of SLUG (Fig. 3B). We therefore tested whether SLUG modulates glioma cell migration. Adenovirus-based overexpression of SLUG induces migration whereas knockdown of SLUG mitigates migration (Fig. 4).

In our microarray data we identified several differentially expressed genes that are involved in the remodeling of the ECM. Therefore, we tested whether matrix metalloproteinases (MMPs), the main players in invasive EMT-like processes and destructors of the ECM, were differentially regulated. Even if there was no regulation of MMPs on mRNA level (data not shown), MMP-2 was downregulated in those sCPE-overexpressing cell lines that harbor MMP-2 (LNT-229 and T98G). With the exception of LN-308 cells, also MT1-MMP/MMP-14 was reduced. It has been described that the net MMP-2 activity correlates with the level of TIMP-2 expression (16). Knowing that under certain conditions TIMP-2 activates MMP-2, we analyzed TIMP-2 expression, demonstrating that TIMP-2 was also downregulated in sCPE-overexpressing LNT-229-, T98G- and Tu-132 cells (Fig. 5). 

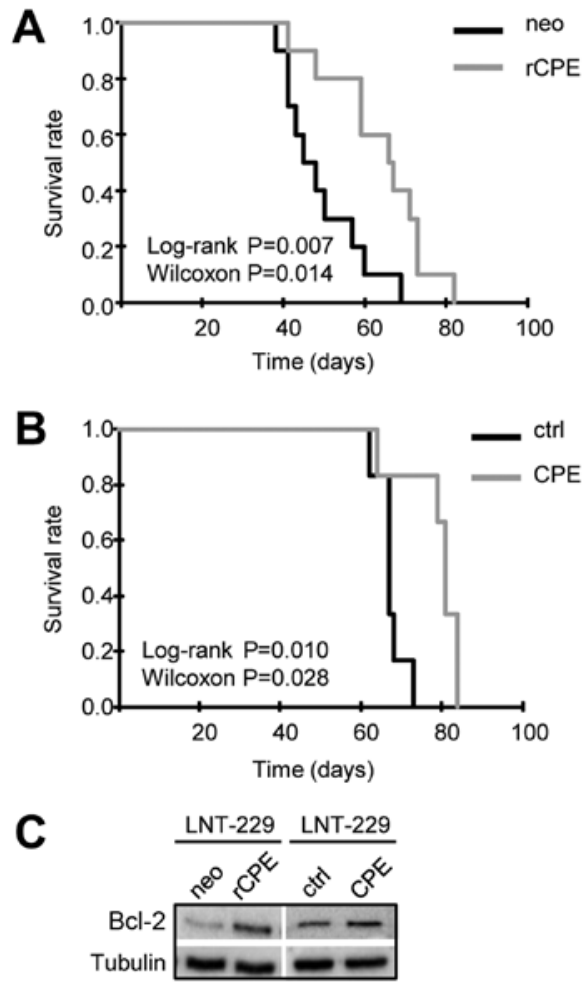

Figure 7. CPE overexpression prolongs the survival of GBM-bearing mice. Survival rate of NMRI nude mice bearing orthotopic tumors derived from CPE-overexpressing and control LNT-229 cells. (A) Survival of tumorbearing mice harboring LNT-229-rCPE or LNT-229-neo control cells (10 mice per group). (B) Survival of tumor-bearing mice harboring LNT229-CPE or LNT-229-ctrl cells (6 mice per group). (C) BCL-2 expression in control (neo, ctrl), rat (left panel) or human (right panel) sCPE overexpressing LNT-229 cells.

SCPE mediated reduction of SLUG and mitigation of glioma cell migration are transmitted via ERK1/2. It has been described that in some cancer cell lines CPE can also transmit its activity via ERK1/2 (17). We analyzed whether ERK phosphorylation was altered in both rat and human SCPE-overexpressing human glioma cells and found elevated ERK phosphorylation in LNT-229 and Tu-132 sCPE-overexpressing cells (Fig. 6A). After inhibition of ERK1/2 activation using the MEK inhibitor U0126 no reduction of SLUG was detectable anymore in sCPE-overexpressing LNT-229 and Tu-132 cells (Fig. 6C). Whereas no significant effect of U0126 on cell migration was detectable in LNT-229 and Tu-132 control cells, addition of U0126 abolished the anti-migratory activity of sCPE in the sCPE-overexpressing cells (Fig. 6D). To determine the upstream cell surface receptor by which extracellular sCPE mediates ERK activation we performed a membrane-based assay that allows the detection of 49 phosphorylated receptor tyrosine kinases. We found a 1.5-fold upregulation of epidermal growth factor receptor (EGFR) phosphorylation (Fig. 6E), however, this could not be confirmed by immunoblot analysis of phospho-EGFR in LNT-229 glioma cells cultivated in sCPE-containing conditioned medium (Fig. 6F and data not shown) indicating that phosphorylation of the EGFR may not be responsible for sCPE-mediated ERK phosphorylation.

SLUG expression can also be regulated by STAT3. Since many of the mRNAs we found to be differentially expressed by microarray expression analysis are associated to STAT3, we also analyzed STAT3 phosphorylation, but did not detect any changes in phospho-STAT3 in sCPE-overexpressing cells (Fig. 6G).

sCPE overexpression prolongs the survival of glioma-bearing mice. In order to consider a possible translational application of sCPE and knowing that CPE, among 311 proteases, is the only peptidase that is downregulated in GBM (18), we were interested whether the anti-migratory effects induced by SCPE we observed in vitro is associated to the survival of glioma-bearing mice. For this we implanted either rat or human sCPE-overexpressing LNT-229 or their sibling control cells into the right striatum of nude mice. The time-point the mice developed neurological symptoms they were sacrificed. At this time-point all mice developed large tumors. Survival analyses demonstrate that mice harboring tumors derived from sCPE-overexpressing cells survived significantly longer than mice harboring control tumors (Fig. 7A and B). Prolonged survival of CPE-tumor bearing mice was not an effect of reduced proliferation in SCPE expressing cells (Fig. 1A, lower panel and data not shown) and even not an effect of elevated apoptosis in these cells since no differences in caspase activity were detectable (7). Besides and due to the data shown by Murthy et al $(8,17)$, who demonstrated that CPE upregulates BCL-2 we analyzed BCL-2 expression in control-and sCPE-overexpressing glioma cells lines and found even enhanced BCL-2 protein expression in all sCPE expressing cell lines (Fig. 7C and data not shown).

\section{Discussion}

The effect of CPE on tumor cell migration has been described for several tumor entities and also for glioma $(7,17)$. We have demonstrated that a secreted form CPE is responsible for its anti-migratory effect in at least three established and two primary glioma cell lines. Primary GBM cells normally grow as neurospheres and at least a subpopulation of these cells provide glioma stem cell characteristics. In our experiments primary GBM cells were grown in the presence of FCS leading to a more differentiated and adherently growing phenotype. However, this allows the comparison of the anti-migratory function of sCPE in adherently growing established and primary cells.

There are hints that CPE modulates the ERK1/2, AKT and WNT signaling pathways $(8,19,20)$, by this also regulating gene expression, however, it still remains unclear whether changes in the activation of these pathways are responsible for reduced glioma cell motility. In sCPE-overexpressing cells we found altered expression of at least 100 genes involved in the regulation of cell motility (data not shown). Among those regulated genes the main part is involved in the regulation of the TGF- $\beta$, Ccd42, PAK, FAK, STAT3 and integrin signaling pathways which we have found to be negatively regulated by sCPE using IPA (Table I and Fig. 2). qRT-PCR validation of assorted genes that are known to regulate cell motility at different stages led to the identification of at least three antimigratory genes significantly upregulated by $\mathrm{sCPE}$ (Fig. 2C). CD9/MRP-1 is closely linked to plateled derived growth factor mediated glioma cell migration (21), downregulation of MGST1 weakens cell adhesion in PC12 cells (22) whereas 
PCDH17 regulates actin dynamics and inhibits migration and invasion $(23,24)$. In contrast, many pro-migratory genes like ADAMTS4, MGAT4A, SPP1, STC1 and SNAI2/SLUG are downregulated by sCPE (Fig. 2C). ADAMTS4 is a matrix degrading protease important for glioma migration (25), STC1, a biomarker of glioma progression, has been described as a hypoxia-dependent migration factor (26), SPP1 is a matricellular protein that promotes glioma cell migration and invasion (27) and MGAT4A is upregulated in several tumors and has been suggested to promote invasion and metastasis (28-31).

One prominent factor we found to be highly downregulated in SCPE-overexpressing glioma cells was the SNAIL family transcriptional regulator SLUG. SLUG is upregulated during the epithelial to mesenchymal transition (EMT) in epithelial cancers and is a prominent metastatic factor (32). GBM is not an epithelial tumor, but EMT-like processes have been described for GBM, and enhanced SLUG expression is linked to the more invasive and migratory mesenchymal phenotype of gliomas (10,33-35). SLUG seemed to be directly regulated by $\mathrm{sCPE}$, since overexpression of $\mathrm{sCPE}$ led to reduced SLUG expression and migration, whilst knockdown of CPE to enhanced SLUG expression and migration (Figs. 2 and 3). SLUG is a highly unstable protein with a half-life of $<1 \mathrm{~h}$ (36). This might explain why the adenoviral mediated upregulation of SLUG did not enhance migration as prominently as the SLUG knockdown reduced migration (Fig. 4). SLUG provides its pro-migratory function by different pathways. Besides, it binds to the promoters of the L1-CAM gene family that augment glioma cell motility and invasion and correlate with FAK activity $(37,38)$. We identified L1-CAM2/CHL1 to be 5.1x downregulated in sCPE-overexpressing cells. MMPs are also involved in EMT-like processes and their expression is regulated by SNAIL family members (39). In glioma, MMPs are mainly regulated by TGF- $\beta$, a pathway we identified to be negatively regulated in sCPE-overexpressing glioma cells (Fig. 2). We found reduced levels of MMP-2, MT1-MMP/MMP-14 and TIMP-2 in those CPE-overexpressing cell that express these enzymes (Fig. 5), suggesting a direct correlation of sCPE, TGF- $\beta$, SLUG and MMPs. In this regard it is known that in mesenchymal, but not in glial areas of brain tumors, SLUG, TGF- $\beta$, MMP-2 and MMP-9 are highly expressed or activated $(35,40)$. Consistently, we found high CPE expression in the more adhesive and lesser migratory epithelial like-cell fraction of a gliosarcoma, whereas the sarcoid fraction of this tumor was negative for CPE (unpublished data). Notably, the level of MMP reduction did not strictly correlate with the level of sCPE-expression, sCPE-mediated reduction of SLUG and mitigation of migration. This indicates that processes such as sCPE-mediated differential expression of other migration modulating genes, influencing sCPE on migratory pathways or even an interaction between these pathways and genes might play pivotal roles in the anti-migratory effect of SCPE.

It has been recently described that ERK1/2 is phosphorylated and activated in CPE overexpressing cells $(17,19)$. Elevated ERK phosphorylation in sCPE-overexpressing glioma cells is paralleled by reduced SLUG expression and migration. Consistently, inhibition of ERK1/2 activation completely abolishes the sCPE-mediated downregulation of SLUG and led to elevated cell migration, indicating that sCPE-dependent SLUG expression and cell motility is regulated via ERK1/2 (Fig. 6). The typical activation of ERK1/2 by EGFR leads to enhanced cell migration in many tumor cells. However, no phosphorylation of EGFR was detectable in sCPE-overexpressing cells or by addition of SCPE-containing supernatants to parental glioma cells (Fig. 6). Besides, inhibition of ERK1/2 activation by U0126 does not alter the migratory potential of several glioma cell lines $(41,42)$ as we have also seen in LNT-229 and Tu-132 control cells. Therefore, we suggest that in glioma cells and in the context of sCPE overexpression, the activation of ERK $1 / 2$ by a yet unknown receptor provides anti-migratory effects whereas under other circumstances when no or only very low amounts of sCPE are present, phosphorylation of ERK1/2 can induce migration. Identification of the signals or factors that determine ERK1/2 to work in a pro- or anti-migratory fashion needs further intensive investigation. It will also be a challenge for the future to identify the upstream factors that led to the observed activation of ERK $1 / 2$ by $\mathrm{sCPE}$.

One possible mechanism how CPE might also mitigate glioma cell migration beside downregulating SLUG is the effect of CPE on the WNT-pathway as described by Skalka et al (43). WNT regulates the architecture of the cytoskeleton and in this regard is responsible for cell shape, cell adhesion and cell motility. In HEK cells, CPE aggregates with and refrains WNT3a in vesicle-like structures, leading to reduced WNT secretion and by this mechanism putatively inhibits WNT3a effects. This assumption and our observation that sCPE negatively regulates Rac1 (unpublished data), might explain why sCPE-overexpression (7), or addition of sCPE-containing supernatants to parental glioma cells (Fig. 1), induce the formation of LFA complexes. To what extent a WNT3a-CPE complex is involved in the reduction of migration in glioma cells needs further investigation. Another study has shown that CPE deposited on the plasma membrane is rapidly internalized and recycled back to the transgolgi network by ARF6 (44). ARF6 is necessary for tumor cell invasion and inhibition of ARF6 reduces cell migration. Therefore, one can hypothesize that most ARF6 will be bound to overexpressed CPE and this way is not able any more to transmit its pro-migratory function (45). However, in the sCPE-overexpressing glioma cells CPE is presented mainly in a secreted extracellular and not in a membrane bound form. Therefore, it is still speculative whether an interaction of CPE and ARF6 in glioma cells occurs and by this interaction CPE reduces ARF6-mediated cell motility.

Using an in vivo orthotopic mouse glioma model we demonstrated that sCPE-overexpression in the growing tumor resulted in a prolonged survival of tumor-bearing mice (Fig. 7), indicating that sCPE-overexpressing glioma cells presenting a lower migratory capacity might not be as malignant as their counterparts (presenting only low or even no CPE expression) that exhibit a highly migratory and invasive potential. In this model we could not completely reason that the prolonged survival is solely a result of the reduced migration we observed in vitro since many pathways are modulated by sCPE-overexpression that also might influence other processes involved in tumor malignancy such as clonogenic survival, TGF- $\beta$-mediated immunosuppression, 
changes in tumor cell metabolism and others. However, prolonged survival of CPE glioma-bearing mice was not caused by sCPE-mediated reduction of proliferation (Fig. 1A) or induction of apoptosis since CPE-overexpressing cells provided no enhanced caspase activity (7), but showed elevated Bcl-2 levels (Fig. 7C).

To conclude, in glioma cells sCPE is a regulator of the expression of motility-associated genes and pathways. Especially SLUG was downregulated by sCPE. By downregulation of SLUG, sCPE at least partially provides its anti-migratory function. There are multiple other signaling cascades and targets by which sCPE might also transmit its anti-migratory capacity. How far and to what extent single pathways, other differentially expressed genes beside SLUG, or the interconnection of SCPE-regulated pathways are involved in the anti-migratory effect of CPE needs further investigation. Last but not least, the expression of CPE in glioma cells resulted into a survival benefit in a mouse glioma model.

\section{Acknowledgements}

The present study was supported by the German Cancer Foundation (Deutsche Krebshilfe) to U.N. (no. 110450) and M.M. (no. 110451).

\section{References}

1. Zhong J, Paul A, Kellie SJ and O'Neill GM: Mesenchymal migration as a therapeutic target in glioblastoma. J Oncol 2010: 430142, 2010.

2. Phillips HS, Kharbanda S, Chen R, Forrest WF, Soriano RH, Wu TD, Misra A, Nigro JM, Colman H, Soroceanu L, et al: Molecular subclasses of high-grade glioma predict prognosis, delineate a pattern of disease progression, and resemble stages in neurogenesis. Cancer Cell 9: 157-173, 2006.

3. Iser IC, Pereira MB, Lenz G and Wink MR: The epithelialto-mesenchymal transition-like process in glioblastoma: An updated systematic review and in silico investigation. Med Res Rev 37: 271-313, 2016.

4. Naumann U, Harter PN, Rubel J, Ilina E, Blank AE, Esteban H and Mittelbronn M: Glioma cell migration and invasion as potential target for novel treatment strategies. Transl Neurosci 4: 314-329, 2013.

5. Cawley NX, Wetsel WC, Murthy SR, Park JJ, Pacak K and Loh YP: New roles of carboxypeptidase $E$ in endocrine and neural function and cancer. Endocr Rev 33: 216-253, 2012.

6. Lee TK, Murthy SR, Cawley NX, Dhanvantari S, Hewitt SM Lou H, Lau T, Ma S, Huynh T, Wesley RA, et al: An N-terminal truncated carboxypeptidase $\mathrm{E}$ splice isoform induces tumor growth and is a biomarker for predicting future metastasis in human cancers. J Clin Invest 121: 880-892, 2011.

7. Höring E, Harter PN, Seznec J, Schittenhelm J, Bühring HJ, Bhattacharyya S, von Hattingen E, Zachskorn C, Mittelbronn M and Naumann U: The 'go or grow' potential of gliomas is linked to the neuropeptide processing enzyme carboxypeptidase $\mathrm{E}$ and mediated by metabolic stress. Acta Neuropathol 124: 83-97, 2012.

8. Murthy SR, Thouennon E, Li WS, Cheng Y, Bhupatkar J, Cawley NX, Lane M, Merchenthaler I and Loh YP: Carboxypeptidase $\mathrm{E}$ protects hippocampal neurons during stress in male mice by up-regulating prosurvival BCL2 protein expression. Endocrinology 154: 3284-3293, 2013.

9. Weiler M, Bähr O, Hohlweg U, Naumann U, Rieger J, Huang H, Tabatabai G, Krell HW, Ohgaki H, Weller M, et al: BCL-xL: Time-dependent dissociation between modulation of apoptosis and invasiveness in human malignant glioma cells. Cell Death Differ 13: 1156-1169, 2006.

10. Yang HW, Menon LG, Black PM, Carroll RS and Johnson MD: SNAI2/Slug promotes growth and invasion in human gliomas. BMC Cancer 10: 301, 2010.
11. Naumann U, Kügler S, Wolburg H, Wick W, Rascher G, Schulz JB, Conseiller E, Bähr M and Weller M: Chimeric tumor suppressor 1, a p53-derived chimeric tumor suppressor gene, kills p53 mutant and p53 wild-type glioma cells in synergy with irradiation and CD95 ligand. Cancer Res 61: 5833-5842, 2001.

12. He TC, Zhou S, da Costa LT, Yu J, Kinzler KW and Vogelstein B: A simplified system for generating recombinant adenoviruses. Proc Natl Acad Sci USA 95: 2509-2514, 1998.

13. Naumann U, Huang H, Wolburg H, Wischhusen J, Weit S, Ohgaki $\mathrm{H}$ and Weller M: PCTAIRE3: A putative mediator of growth arrest and death induced by CTS-1, a dominant-positive p53-derived synthetic tumor suppressor, in human malignant glioma cells. Cancer Gene Ther 13: 469-478, 2006.

14. Benjamini Y and Hochberg Y: Controlling the false discovery rate: A practical and powerfull approach to multiple testing. J R Stat Soc B 57: 289-300, 1995.

15. Giese A, Loo MA, Tran N, Haskett D, Coons SW and Berens ME: Dichotomy of astrocytoma migration and proliferation. Int $\mathrm{J}$ Cancer 67: 275-282, 1996.

16. Bernardo MM and Fridman R: TIMP-2 (tissue inhibitor of metalloproteinase-2) regulates MMP-2 (matrix metalloproteinase-2) activity in the extracellular environment after pro-MMP-2 activation by MT1 (membrane type 1)-MMP. Biochem J 374: 739-745, 2003.

17. Murthy SR, Dupart E, Al-Sweel N, Chen A, Cawley NX and Loh YP: Carboxypeptidase E promotes cancer cell survival, but inhibits migration and invasion. Cancer Lett 341: 204-213, 2013.

18. Verbovšek U, Motaln H, Rotter A, Atai NA, Gruden K, Van Noorden CJ and Lah TT: Expression analysis of all protease genes reveals cathepsin $\mathrm{K}$ to be overexpressed in glioblastoma. PLoS One 9: e111819, 2014.

19. Cheng Y, Cawley NX and Loh YP: Carboxypeptidase E/NFa1: A new neurotrophic factor against oxidative stress-induced apoptotic cell death mediated by ERK and PI3-K/AKT pathways. PLoS One 8: e71578, 2013.

20. Skalka N, Caspi M, Caspi E, Loh YP and Rosin-Arbesfeld R: Carboxypeptidase E: A negative regulator of the canonical Wnt signaling pathway. Oncogene 32: 2836-2847, 2013.

21. Jeibmann A, Halama K, Witte HT, Kim SN, Eikmeier K, Koos B, Klämbt $\mathrm{C}$ and Paulus W: Involvement of CD9 and PDGFR in migration is evolutionarily conserved from Drosophila glia to human glioma. J Neurooncol 124: 373-383, 2015.

22. Sobczak M, Boczek T, Ferenc B, Taha J, Kozaczuk A, Wiktorska M, Sacewicz-Hofman I, Niewiarowska J and Zylinska L: Functional characteristic of PC12 cells with reduced microsomal glutathione transferase 1. Acta Biochim Pol 57: 589-596, 2010

23. Haruki S, Imoto I, Kozaki K, Matsui T, Kawachi H, Komatsu S, Muramatsu T, Shimada Y, Kawano T and Inazawa J: Frequent silencing of protocadherin 17, a candidate tumour suppressor for esophageal squamous cell carcinoma. Carcinogenesis 31 : 1027-1036, 2010

24. Dang Z, Shangguan J, Zhang C, Hu P, Ren Y, Lv Z, Xiang H and Wang X: Loss of protocadherin-17 (PCDH-17) promotes metastasis and invasion through hyperactivation of EGFR/MEK/ERK signaling pathway in hepatocellular carcinoma. Tumour Biol 37: 2527-2535, 2016

25. Held-Feindt J, Paredes EB, Blömer U, Seidenbecher C, Stark AM, Mehdorn HM and Mentlein R: Matrix-degrading proteases ADAMTS4 and ADAMTS5 (disintegrins and metalloproteinases with thrombospondin motifs 4 and 5) are expressed in human glioblastomas. Int J Cancer 118: 55-61, 2006.

26. Lee JK, Joo KM, Lee J, Yoon Y and Nam DH: Targeting the epithelial to mesenchymal transition in glioblastoma: The emerging role of MET signaling. Onco Targets Ther 7: 1933-1944, 2014.

27. Lu DY, Yeh WL, Huang SM, Tang CH, Lin HY and Chou SJ: Osteopontin increases heme oxygenase-1 expression and subsequently induces cell migration and invasion in glioma cells. Neuro-oncol 14: 1367-1378, 2012.

28. Potapenko IO, Haakensen VD, Lüders T, Helland A, Bukholm I, Sørlie T, Kristensen VN, Lingjaerde OC and Børresen-Dale AL: Glycan gene expression signatures in normal and malignant breast tissue; possible role in diagnosis and progression. Mol Oncol 4: 98-118, 2010.

29. Niimi K, Yamamoto E, Fujiwara S, Shinjo K, Kotani T, Umezu T, Kajiyama H, Shibata K, Ino K and Kikkawa F: High expression of $\mathrm{N}$-acetylglucosaminyltransferase IVa promotes invasion of choriocarcinoma. Br J Cancer 107: 1969-1977, 2012 
30. Fan J, Wang S, Yu S, He J, Zheng W and Zhang J: N-acetylglucosaminyltransferase IVa regulates metastatic potential of mouse hepatocarcinoma cells through glycosylation of CD147. Glycoconj J 29: 323-334, 2012.

31. Ide Y, Miyoshi E, Nakagawa T, Gu J, Tanemura M, Nishida T, Ito T, Yamamoto H, Kozutsumi Y and Taniguchi N: Aberrant expression of $\mathrm{N}$-acetylglucosaminyltransferase-IVa and $\mathrm{IVb}$ (GnT-IVa and b) in pancreatic cancer. Biochem Biophys Res Commun 341: 478-482, 2006.

32. Tania M, Khan MA and Fu J: Epithelial to mesenchymal transition inducing transcription factors and metastatic cancer. Tumour Biol 35: 7335-7342, 2014

33. Kahlert UD, Nikkhah G and Maciaczyk J: Epithelial-tomesenchymal(-like) transition as a relevant molecular event in malignant gliomas. Cancer Lett 331: 131-138, 2013.

34. Chesnelong $\mathrm{C}$ and Luchman AH: STAT3 is a key regulator of an 'EMT-like' process mediated by Slug in GBM. Cancer Res 76: 2524,2016

35. Iwadate Y: Epithelial-mesenchymal transition in glioblastoma progression. Oncol Lett 11: 1615-1620, 2016.

36. Shih JY and Yang PC: The EMT regulator slug and lung carcinogenesis. Carcinogenesis 32: 1299-1304, 2011.

37. Geismann C, Arlt A, Bauer I, Pfeifer M, Schirmer U, Altevogt P, Müerköster SS and Schäfer H: Binding of the transcription factor Slug to the L1CAM promoter is essential for transforming growth factor- $\beta 1$ (TGF- $\beta$ )-induced L1CAM expression in human pancreatic ductal adenocarcinoma cells. Int J Oncol 38: 257-266, 2011.

38. Yang M, Li Y, Chilukuri K, Brady OA, Boulos MI, Kappes JC and Galileo DS: L1 stimulation of human glioma cell motility correlates with FAK activation. J Neurooncol 105: 27-44, 2011.
39. Lamouille S, Xu J and Derynck R: Molecular mechanisms of epithelial-mesenchymal transition. Nat Rev Mol Cell Biol 15: 178-196, 2014.

40. Nagaishi M, Paulus W, Brokinkel B, Vital A, Tanaka Y, Nakazato Y, Giangaspero F and Ohgaki H: Transcriptional factors for epithelial-mesenchymal transition are associated with mesenchymal differentiation in gliosarcoma. Brain Pathol 22: 670-676, 2012.

41. Goldberg L and Kloog Y: A Ras inhibitor tilts the balance between Rac and Rho and blocks phosphatidylinositol 3-kinase-dependent glioblastoma cell migration. Cancer Res 66: 11709-11717, 2006.

42. Stepanenko AA, Andreieva SV, Korets KV, Mykytenko DO, Baklaushev VP, Chekhonin VP and Dmitrenko VV: mTOR inhibitor temsirolimus and MEK1/2 inhibitor U0126 promote chromosomal instability and cell type-dependent phenotype changes of glioblastoma cells. Gene 579: 58-68, 2016.

43. Skalka N, Caspi M, Lahav-Ariel L, Loh YP, Hirschberg K, Rosin-Arbesfeld R and Carboxypeptidase E: Carboxypeptidase $\mathrm{E}(\mathrm{CPE})$ inhibits the secretion and activity of Wnt3a. Oncogene 35: 6416-6428, 2016.

44. Arnaoutova I, Jackson CL, Al-Awar OS, Donaldson JG and Loh YP: Recycling of Raft-associated prohormone sorting receptor carboxypeptidase $\mathrm{E}$ requires interaction with ARF6. Mol Biol Cell 14: 4448-4457, 2003.

45. Sabe H: Requirement for Arf6 in cell adhesion, migration, and cancer cell invasion. J Biochem 134: 485-489, 2003. 